\title{
Improvement of Hen Egg White lysozyme Crystal Quality by Control of Dehydration Process
}

\author{
Haruhiko Koizumi, * Satoshi Uda, Katsuo Tsukamoto, Kenji Hanada, Ryo Suzuki, \\ Masaru Tachibana, and Kenichi Kojima \\ E-mail: h_koizumi@sporr.mie-u.ac.jp
}

Supporting Information

List of contents

Movie S1: Series of X-ray topographic images 
Movie S1: Series of X-ray topographic images

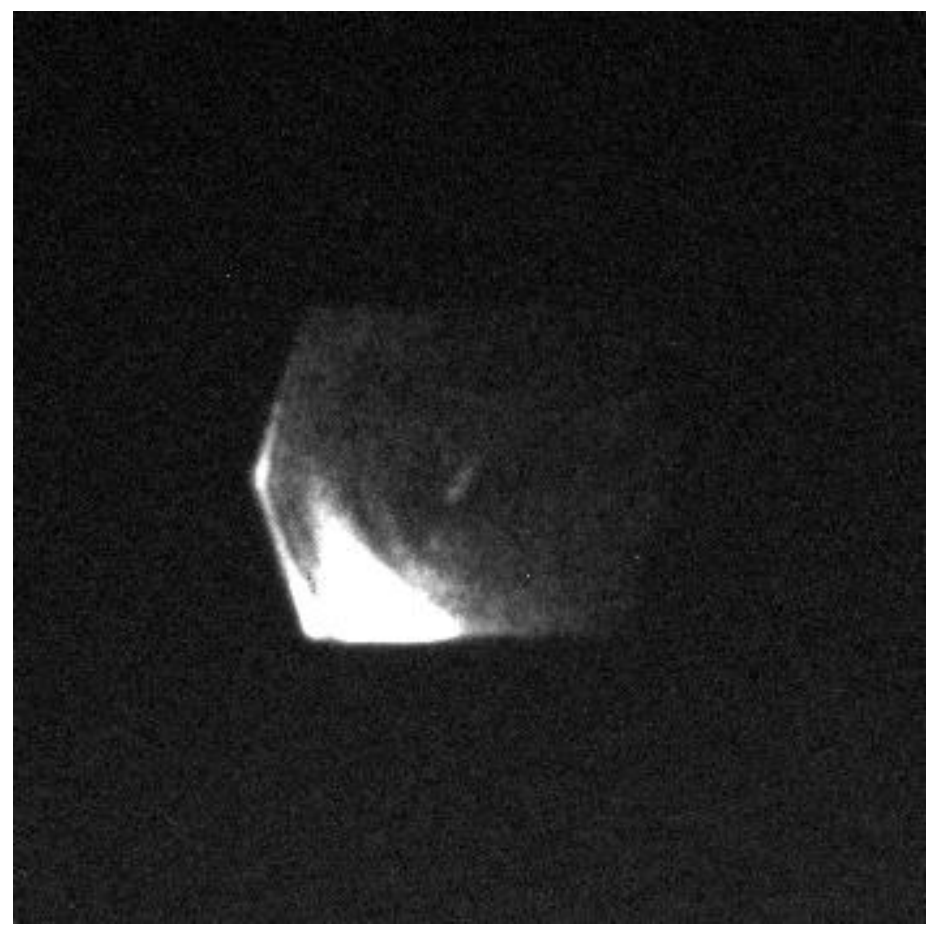

A series of X-ray topographic images obtained using the same crystal as that used for $\mathrm{X}$-ray topography. The topographic images were taken using the 110 reflection from the HEW lysozyme crystal grown with a $\mathrm{NaCl}$ concentration of $1.20 \mathrm{~mol} / \mathrm{L}$. 